\title{
ERRATA
}

\section{Semi-Empirical Model using Radiant Coal Power to Predict Cigarette Ignition Strength as Measured by Extinction Test}

\author{
Tim S. Sherwood, Jason C. Issac, Ram M. Murthi, Karl E. Wiedemann, \\ Srinivasan Janardhan and Justin S. Jones
}

\begin{abstract}
Upon reviewing their article, the authors noted several errors that were missed in the preparation and peer-review process as well as several points for clarification. These errors were included in both the print and online versions of the original article. The corrections and clarifications are:
\end{abstract}

\section{Corrections:}

1. Page $238,2^{\text {nd }}$ full paragraph, $2^{\text {nd }}$ sentence: Change " $[5]$ " to " $[8]$ ".

2. Page $238,2^{\text {nd }}$ full paragraph, last sentence: Change " $4 \mathrm{~mm}^{2}$ " to " $0.25 \mathrm{~mm}^{2}$ ".

3. Page $239,2^{\text {nd }}$ line after Equation 2: Change " $4 \times 10^{-6} \mathrm{~m}^{2}$ " to " $0.25 \times 10^{-6} \mathrm{~m}^{2}$ ".

4. Page 239, Model Development and Results, $1^{\text {st }}$ full paragraph, $2^{\text {nd }}$ sentence: Change "5.6-8.1 mm" to "5.4-7.9 mm".

5. Page 239, Model Development and Results, $1^{\text {st }}$ full paragraph, $2^{\text {nd }}$ sentence: Change "21.7 mm" to "22.7 mm".

6. Page 244: Change Equation 5 to: $E_{T}=A_{1}+\frac{\left(A_{2}-A_{1}\right)}{\left(1+e^{\frac{\left(P_{C}-x_{o}\right)}{d x}}\right)}$

7. Page 244, $1^{\text {st }}$ line after Equation 5: Change "lower and upper" to "upper and lower".

\section{Clarifications:}

1. Page $237,3^{\text {rd }}$ paragraph, $1^{\text {st }}$ sentence: Insert " $m$ " after " $\mu$ ".

2. Page $237,3^{\text {rd }}$ paragraph, $4^{\text {th }}$ sentence: Change sentence to read: "The acceptance angle for a pixel at the center of the field of view and at the edge of the long axis of the field of view differs by less than $10 \%$, which is equivalent to a length of approximately $0.03 \mathrm{~mm} . "$

3. Page $238,3^{\text {rd }}$ paragraph, $1^{\text {st }}$ sentence: Insert “ $x(t)$ " between "position" and "( $\left.\mathrm{mm}\right)$ ". 
4. Page 238: Change Equation 1 to: $x(t)=\frac{\sum_{i=1}^{k} x_{i} T_{i}}{\sum_{i=1}^{k} T_{i}}, k \neq 0$

5. Page 239: Change Equation 2 to: $P(t)=\sigma \varepsilon A \sum_{i=1}^{k} T_{i}^{4}, k \neq 0$

6. page $245,1^{\text {st }}$ full paragraph, $1^{\text {st }}$ sentence: Insert "ed" after "predict".

7. Page $247,1^{\text {st }}$ full paragraph, $4^{\text {th }}$ sentence: Change "various" to "the designated".

The authors regret any inconvenience to the readers.

The online version of the original article can be found at http://dx.doi.org/10.1007/s10694-006-7507-8 